\title{
ARE PIXEL INTENSITIES PROPORTIONAL TO RADAR CROSS SECTION IN SAR IMAGES?
}

\author{
Björn J. Döring and Marco Schwerdt \\ Microwaves and Radar Institute \\ German Aerospace Center (DLR) \\ Oberpfaffenhofen, Germany
}

\section{INTRODUCTION}

Radar cross section has long been the measurement quantity of choice to describe the radar reflectivity of point targets in calibrated synthetic aperture radar (SAR) images [1, $2,3,4]$. Derived quantities like the backscatter coefficient are equally in wide-spread use to radiometrically describe, in scientific applications often even more relevant, distributed targets. This paper now raises the question if radar cross section (RCS) really is the best choice to link pixel intensities or digital numbers to a physical unit. In fact, in Sec. 2 it is shown that RCS actually is not a good choice out of two major reasons, both of which result from the RCS being frequency dependent.

The authors propose to distinguish the radar cross section, a body property, from the actual radiometric measurement quantity in SAR images by introducing the dedicated term equivalent RCS, described in more detail in Sec. 3. The implications for measurements and radiometric calibration are described in Sec. 4.

\section{CRITICISMS ON USING RCS AS THE RADIOMETRIC MEASUREMENT QUANTITY}

The radar cross section $\sigma$ of any target is defined as the scaled ratio of the scattered power (seen at distance $R$ away from the target) to the incident power, as in

$$
\sigma=\lim _{R \rightarrow \infty} 4 \pi R^{2} \frac{\left|\mathbf{E}_{s}\right|^{2}}{\left|\mathbf{E}_{i}\right|^{2}}
$$

where $\mathbf{E}_{s}$ and $\mathbf{E}_{i}$ are the scattered and incident electrical fields [5].

The fundamental problem of using RCS as the output measurement quantity for describing pixel intensities in SAR images is rooted in the underlying, often only implicitly stated, measurement model. In fact, the pixel intensities in SAR images are only linked to target RCS if the target frequency transfer function $H_{t}$ can be modeled as

$$
H_{t}(j \omega)=\text { const }
$$

within the bandwidth of the range signal. Whereas this is a practical and justified approximation for many natural targets observed by a sufficiently narrow-band SAR systems, it does not hold for all (and especially wideband) cases. Generally, targets should rather be modeled by

$$
H_{t}(j \omega)=A(\omega) e^{j \varphi(\omega)}
$$

where $A(\omega)$ and $\varphi(\omega)$ are the frequency dependent gain and phase functions, respectively. The simplified measurement model in Eq. (2) is inherently included in the more general definition of Eq. (3).

By acknowledging the measurement model of Eq. (3), the first criticism arises. If the RCS of a target varies significantly over the relevant range bandwidth, no single RCS can be attached to the measurement. The pixel intensities after SAR focusing are rather the result of a weighted average over all frequency-dependent target RCS values.

The second criticism arises due to the filtering operation during SAR processing. If the complex signal which is 
recorded by the SAR instrument is denoted as $s_{r}$ then

$$
s_{\text {out }}(t)=\int_{-\infty}^{+\infty} s_{r}(u) h^{*}(u-t) d u
$$

where $s_{\text {out }}$ is the complex filter output; the filter $h$ is chosen to be the transmit signal replica, which is often weighted for better sidelobe suppression [4]. Taking the measurement model of Eq. (3) into account, it becomes apparent that $h$ is unmatched in a target-specific manner if the received signal is not simply a time-shifted and amplitude-scaled version of the transmit signal (which would result from Eq. (2)). This targetdependent unmatched filtering leads, of course, to a pixel intensity which is not proportional to RCS anymore. In other words, the SAR processing considers complex signal amplitudes but the definition of RCS in Eq. (1) depends on signal magnitudes only so they can never be fully proportional.

\section{PROPOSED NEW RADIOMETRIC MEASUREMENT QUANTITY TERMINOLOGY}

It was argued that, in the general case, the pixel intensities in SAR images are not proportional to the target radar cross section $\sigma$ or derived quantities like the backscattering coefficient $\sigma^{0}$. In other words, the measurement quantity in radiometric SAR measurements is not RCS, and therefore it should not be named RCS.

It is proposed to instead call the measurement quantity for point targets equivalent radar cross section. The equivalent radar cross section $\sigma_{e}$ shall be equal to the radar cross section of a perfectly conducting sphere which would result in an equivalent pixel intensity if the sphere were to replace the measured target. The definition exploits the crucial frequency independence of the RCS of a sphere with radius $a$

$$
\sigma_{\text {sphere }}=\pi a^{2}
$$

which is valid as long as the sphere circumference is much (say, at least more than ten times) larger than the wavelength [5].

Replacing RCS by equivalent RCS pays tribute to the two general points of critique. Now,

- the filtering of complex signals according to Eq. (4) is correctly distinguished from the definition of RCS, which only takes signal magnitudes into consideration, and

- cases for which the target frequency dependence is significant according to Eq. (3) are covered.

One could say that the terminology of equivalent RCS allows to distinguish between the target RCS (Eq. (1)) and the target's pixel intensity as seen through the eyes of the SAR processing filter (Eq. (4)). The proposed terminology allows to describe target backscatters with an arbitrary frequency dependence according to Eq. (3), including targets with a flat frequency response. Depending on the target, the measurement uncertainty can appear greatly reduced due to the more accurate measurement model, which especially benefits high bandwidth, high accuracy systems.

The transition from the present to the proposed terminology is smooth. For instance, the measurement unit for RCS and equivalent RCS is the same: square meter. Also, it is straightforward to transform the backscatter coefficient $\sigma^{0}$ and other derived quantities to equivalent quantities, i. e., to an equivalent backscatter coefficient $\sigma_{e}^{0}$, etc. Furthermore, describing the measurement quantity in terms of an equivalent physical object (a sphere) allows one to form a simple mental model of what this quantity means.

\section{IMPLICATIONS FOR MEASUREMENTS AND RADIOMETRIC CALIBRATION}

Embracing rather than neglecting the frequency dependent radar backscatter is the main mental shift which follows from replacing RCS by equivalent $R C S$ as the radiometric measurement quantity. The distinction between backscatter magnitudes at discreet frequencies and the target reflectivity as seen by a SAR system is especially important for accurate wideband systems. Whereas first spaceborne sensors like the SIR$\mathrm{C} / \mathrm{X}-\mathrm{SAR}$ instrument still had a relative bandwidth below $1 \%$, nowadays ultra-wideband systems with a relative bandwidth above $100 \%$ are already in use [7]. The large $(>1 \mathrm{~dB})$ radiometric variation over frequency for two exemplary terrain types and several exemplary relative bandwidths is shown in Fig. 1. Introducing equivalent $R C S$ as the new terminology avoids an ambiguity by distinguishing the body property from the quantity that is seen in a SAR image. 


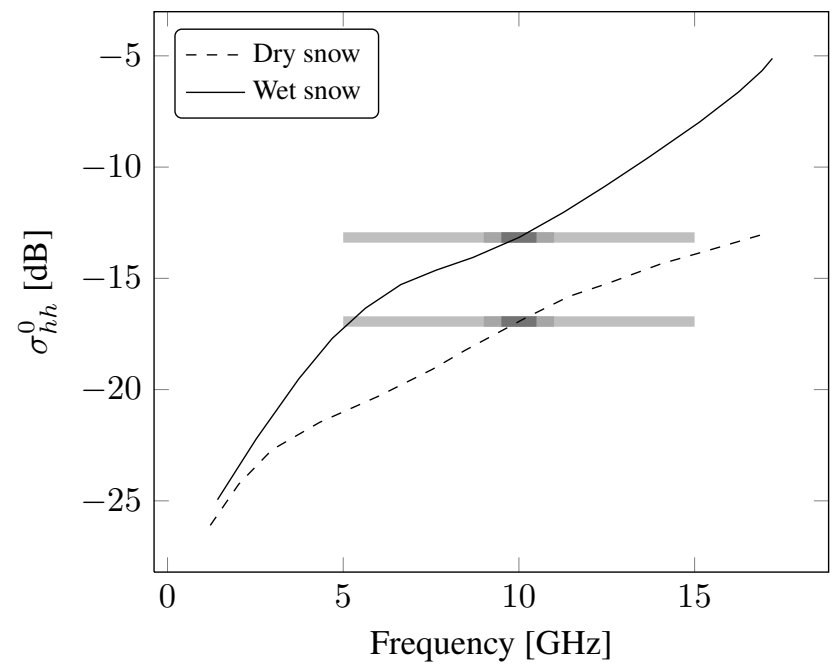

Fig. 1. Frequency dependent backscattering coefficient $\sigma^{0}$ for an exemplary terrain type: dry and wet snow (measured at $\mathrm{HH}$ polarization and an incidence angle of $50^{\circ}$, data from [6]). For illustration purposes, fractional bandwidths of $(10,20$, 100) $\%$ are shown at $10 \mathrm{GHz}$ to emphasize the large frequency dependence within these spectra.

In terms of calibration, the frequency dependent backscatter of man-made reference targets (according to Eq. (3)), which are used to achieve an absolute radiometric calibration of a spaceborne SAR system, will have to be considered in the future. One approach was described in detail in [8], where the known frequency dependence of a reference target is corrected based on a point-target SAR simulation.

\section{CONCLUSION}

This paper discussed the problems associated with denoting RCS as the radiometric measurement quantity in SAR images. The root cause was identified to be the principal frequency dependence of the target backscatter. It was proposed to replace RCS by equivalent RCS as the radiometric measurement quantity in SAR images because a SAR system does not measure RCS and therefore another terminology should be used to describe the measurement quantity.

The implication of the new terminology for measurements was identified to lie in a mental shift toward embracing the frequency dependent target backscatter as a desired feature. In terms of external absolute radiometric calibration, on the other hand, the new terminology asks for a compensation of the frequency dependent backscatter of reference targets.
By adapting equivalent $R C S$ as the measurement quantity, calibration and measurement results become truly compatible across current and future narrow and especially high-accuracy wideband SAR systems.

\section{REFERENCES}

[1] Anthony Freeman, "SAR calibration: An overview," IEEE Transactions on Geoscience and Remote Sensing, vol. 30, no. 6, pp. 1107-1121, Nov. 1992.

[2] John C. Curlander, Synthetic aperture radar: Systems and signal processing, John Wiley \& Sons, Inc., 1991.

[3] Chris Oliver and Shaun Quegan, Understanding synthetic aperture radar images, SciTech Publishing, 2004.

[4] Ian G. Cumming, Digital Processing Of Synthetic Aperture Radar Data: Algorithms And Implementation, Artech House Publishers, 2005.

[5] Eugene F. Knott, John F. Shaeffer, and Michael T. Tuley, Radar cross section, SciTech Publishing, Raleigh, NC, 2 edition, 2004.

[6] Fawwaz T. Ulaby, Handbook of radar scattering statistics for terrain, Artech House, 1989.

[7] Björn Ulander, Lars M. H.and Flood, Per-Olov Frölind, Tommy Jonsson, Anders Gustavsson, Johan Rasmusson, Gunnar Stenström, Arnold Barmettler, and Erich Meier, "Bistatic experiment with ultra-wideband VHFband synthetic-aperture radar," European Conference on Synthetic Aperture Radar, June 2008.

[8] Björn J. Döring, Philipp Looser, Matthias Jirousek, and Marco Schwerdt, "Reference target correction based on point target SAR simulation," IEEE Transactions on Geoscience and Remote Sensing, vol. 50, no. 3, Mar. 2012. 